\title{
Molecular Typing of Bois Noir Phytoplasma Strains in the Chianti Classico Area (Tuscany, Central Italy) and Their Association with Symptom Severity in Vitis vinifera 'Sangiovese'
}

\author{
R. Pierro, A. Passera, A. Panattoni, P. Casati, A. Luvisi, D. Rizzo, P. A. Bianco, F. Quaglino, ${ }^{\dagger}$ and A. Materazzi
}

First, third, fifth, and ninth authors: Department of Agriculture, Food and Environment, University of Pisa, via del Borghetto 80, 56124 Pisa, Italy; second, fourth, seventh, and eighth authors: Department of Agricultural and Environmental Sciences-Production, Landscape, Agroenergy, University of Milan, via Celoria 2, 20133 Milano, Italy; fifth author: Department of Biological and Environmental Sciences and Technologies-University of Salento, via Provinciale Monteroni, 73100 Lecce, Italy; and sixth author: Regional Phytosanitary Service, Laboratory of Phytopathological Diagnostics and Molecular Biology, via Ciliegiole 99, 51100 Pistoia, Italy.

Accepted for publication 11 October 2017.

\begin{abstract}
Bois noir $(\mathrm{BN})$ is the most widespread disease of the grapevine yellows complex in the Euro-Mediterranean area. BN is caused by 'Candidatus Phytoplasma solani' (BNp), transmitted from herbaceous plants to grapevine by polyphagous insect vectors. In this study, genetic diversity among BNp strains and their prevalence and possible association with grapevine symptom severity were investigated in a Sangiovese clone organic vineyard in the Chianti Classico area (Tuscany). Field surveys over 2 years revealed a range of symptom severity on grapevine and an increase of $\mathrm{BN}$ incidence. A TaqMan allelic discrimination assay detected only tufB type $\mathrm{b}$ among BNp strains, suggesting the prevalence of the bindweed-related ecology. Nucleotide sequence analyses of vmpl and stamp genes identified $12 \mathrm{vmpl}$
\end{abstract}

ABSTRACT and 16 stamp sequence variants, showing an overall positive selection for such genes. The prevalent genotype was $\mathrm{Vm} 43 / \mathrm{St} 10$, reported for the first time in this study and closely related to strains identified only in the French Eastern Pyrenees. BNp strains identified in the examined vineyard and mostly grouped in separate bindweed-related phylogenetic clusters showed statistically significant differences in their distribution in grapevines exhibiting distinct symptom severity. These results suggest the possible occurrence of a range of virulence within BNp strain populations in the Chianti Classico area.

Additional keywords: membrane protein, multiple gene typing.
Grapevine yellows (GY) are phytoplasma-associated diseases occurring worldwide in the main grapevine-growing areas. Among $\mathrm{GY}$, bois noir $(\mathrm{BN})$ is one of the most important diseases, responsible for serious crop losses in the Euro-Mediterranean area and in several other countries (Belli et al. 2010). The causal agent of BN was identified in phytoplasma (BNp) strains belonging to the species 'Candidatus Phytoplasma solani', 16SrXII-A subgroup (Quaglino et al. 2013). Disease symptoms consist of plant decline, shriveled grapes, leaf rolling, desiccation of inflorescences, irregular ripening of wood, and reddening or yellowing of leaves on red and white cultivars, respectively (Belli et al. 2010). BN is mainly transmitted to grapevine (Vitis vinifera L.) by the cixiid planthopper Hyalesthes obsoletus Signoret (Sharon et al. 2005), which has a biological cycle that is completed preferentially on field bindweed (Convolvulus arvensis L.) and common nettle (Urtica dioica L.). In fact, infected grapevine is considered a "dead-end host" of BNp because its main vector feeds on this species only occasionally, reducing its role in the disease epidemiology. BN spreads also in areas where H. obsoletus, the main vector, does not occur (Belli et al. 2010; Maixner 2011), and recent studies have proven that Reptalus panzeri Löw and $R$. quinquecostatus Dufour can act as vectors of BNp to grapevine in Serbian and French vineyards, respectively (Cvrković et al. 2014; Chuche et al. 2016). Moreover, other studies have also identified several herbaceous plant hosts playing a direct role in $\mathrm{BN}$ diffusion (Berger et al. 2009; Marchi et al. 2015; Mori et al. 2015).

†Corresponding author: F. Quaglino; E-mail: fabio.quaglino@unimi.it

*The $\boldsymbol{e}$-Xtra logo stands for "electronic extra" and indicates that two supplementary figures and six supplementary tables are published online.

(C) 2018 The American Phytopathological Society
Such evidence reporting the presence of multiple BNp plant and insect hosts in the Euro-Mediterranean area indicates that this phytoplasma exists in varied ecosystems, where selection conceivably alters strain populations (Quaglino et al. 2013, 2017). This hypothesis implies that ecological relationships of $\mathrm{BNp}$ and $\mathrm{BNp}-$ related strains can be reflected in intraspecies strain diversity. Thus, in recent years, numerous studies focused on distinguishing genetic structure of BNp strains with the aim to identify strain-specific molecular markers associated with distinct biological features. Such studies were carried out by nucleotide sequence analyses of the genes tufB, encoding the translation elongation factor Tu (Schneider et al. 1997); secY, encoding a translocation protein (Fialová et al. 2009); and vmpl and stamp, encoding membrane proteins presumably involved in the interaction between $\mathrm{BNp}$ and its hosts (Cimerman et al. 2009; Fabre et al. 2011).

Information obtained from the tufB gene characterization allowed the identification of two main $\mathrm{BNp}$ tufB types associated with herbaceous plant hosts related to distinct epidemiological systems in Europe (Belli et al. 2010; Langer and Maixner 2004; Maixner 2011): (i) tufB type a, associated with $U$. dioica and prevalent in western Europe and northern Italy (Quaglino et al. 2013) and (ii) tufB type b, mainly associated with $C$. arvensis and many other herbaceous hosts and prevalent in central-southern Italy (Murolo and Romanazzi 2016; Pacifico et al. 2009). Furthermore, the analyses of nucleotide sequences of the genes secY, stamp, and vmpl showed a larger variability among BNp strains within the two main tufB types (Foissac et al. 2013; Kostadinovska et al. 2014; Murolo and Romanazzi 2015). Currently, $23 \mathrm{~V}$ types distinguished by $v m p 1$ restriction fragment length polymorphism (RFLP) profiles (nomenclature determined according to SEE-ERANET; X. Foissac, INRA, Bordeaux, France), 63 nucleotide sequence variants for gene $v m p l$, and 35 variants for gene stamp were described among $\mathrm{BNp}$ and ' $\mathrm{Ca}$. P. solani' strains (Murolo and 
Romanazzi 2015; Quaglino et al. 2016). Utilization of molecular markers identified in these studies increased the knowledge of BNp strain movement and host range (Cvrković et al. 2014; Kosovac et al. 2015), and confirmed the presence of a positive selective pressure determining the $\mathrm{BNp}$ strain population complexity in different vineyard agroecosystems (Murolo et al. 2014). Moreover, recent evidence reinforced the hypothesis that $\mathrm{BNp}$ strains, distinguishable using such molecular markers, can exhibit a range of virulence associated with different symptom severity in infected grapevine plants (Quaglino et al. 2016).

The main objectives of this study, carried out over two consecutive seasons in a Sangiovese clone organic vineyard in the Chianti Classico area (Greve in Chianti, Tuscany, central Italy), were to investigate the genetic diversity among $\mathrm{BNp}$ strains through multiple gene typing and to evaluate their possible association with grapevine symptom severity by statistical analyses.

\section{MATERIALS AND METHODS}

Vineyard statement. The study was conducted within an experimental area (735 plants) selected as representative of a vineyard planted in 1997 in Greve in Chianti (FI), located in the traditional grapevine-growing region of Chianti Classico, Tuscany (central Italy) $\left(43^{\circ} 33^{\prime} 21^{\prime \prime} \mathrm{N}, 11^{\circ} 18^{\prime} 8^{\prime \prime} \mathrm{E}\right.$, and $460 \mathrm{~m}$ above sea level). The vineyard was bordered by other vineyards and forests and was conducted according to organic management. Vines ( $V$. vinifera L. 'Sangiovese', I-SS F9 A5 48) were trained as cordon (planting density $2.3 \mathrm{~m}$ between the rows and $0.8 \mathrm{~m}$ along the row).

Symptom observations and plant samples. In 2015 and 2016, each vine was visually assessed for the presence of GY disease, once in June and once in September of each year. The severity of symptoms was classified and sorted according to a GY symptomatic scale from 0 to 3, modified from the one proposed by Riedle-Bauer et al. (2010) as follows: (i) symptom severity class $0=$ healthy plants with no symptoms, (ii) symptom severity class $1=$ one shoot with mild leaf symptoms, (iii) symptom severity class $2=$ two to three shoots with leaf symptoms, and (iv) symptom severity class $3=$ more than three shoots with leaf symptoms and berry shrivel. Furthermore, overall disease severity $(\mathrm{OS})$ in the vineyard was evaluated using the formula $S=\Sigma(c \cdot f) / n$, proposed by Murolo and Romanazzi (2015), where $S$ is the disease severity, $c$ is the symptom severity class, $f$ is the frequency of the symptom severity class, and $n$ is the number of symptomatic plants. For each year, 10 leaves were sampled from each symptomatic plant and from 10 randomly selected symptomless plants for phytoplasma detection and characterization.

$V$. vinifera 'Sangiovese', obtained from the screenhouse of the Department of Agriculture, Food and Environment (University of Pisa, Italy), was used as the healthy control, and $V$. vinifera plants, previously found infected by either ' $\mathrm{Ca}$. P. solani' (subgroup 16SrXIIA) or Flavescence dorée phytoplasmas (FDp) (subgroups 16SrV-C or -D), were used as the infected reference control (IC).

Fresh central midribs of fully developed leaves were dissected and stored at $-20^{\circ} \mathrm{C}$ until DNA extraction.

DNA extraction. DNA was extracted with cetyltrimethylammonium bromide (CTAB)-based buffer from leaf veins according to the protocol described by Li et al. (2008), with some modifications. Briefly, leaf veins $(1 \mathrm{~g})$ were homogenized in plastic bags (Bioreba) with $7 \mathrm{ml}$ of $2 \%$ CTAB buffer using Homex 6 (Bioreba). The homogenate was incubated at $65^{\circ} \mathrm{C}$ for $15 \mathrm{~min}$. DNA was extracted by one volume of chloroform:iso-amylalcohol (24:1) and precipitated with one volume of isopropanol. Pellets were washed with $70 \%$ ethanol, air dried, suspended in $100 \mu \mathrm{l}$ of deionized water, and stored at $-20^{\circ} \mathrm{C}$ until use.

GY phytoplasma detection and relative quantification. Specific detection of phytoplasmas associated with BN and FD, the GY diseases more commonly present in Italy, was carried out by amplification of $16 \mathrm{~S}$ ribosomal DNA (rDNA) through a TaqMan assay using the G8830A AriaMx real-time polymerase chain reaction (PCR) (Agilent Technologies), following reaction conditions as described by Angelini et al. (2007). The template used in the assay was a 1:10 dilution of the DNA extracted from the samples. The grapevine chloroplast chaperonin 21 gene and the DNA extracted from healthy control plants and IC were used as endogenous, negative, and positive controls, respectively. Threshold cycle $(\mathrm{Ct})<$ 37 was associated with the presence of GY phytoplasmas (Mori et al. 2015). As in previous studies (Baric 2012; Minguzzi et al. 2016), the relative quantification of phytoplasmas in each sample was calculated using the following formula: $\Delta \mathrm{Ct}=\mathrm{Ct}_{\mathrm{p}}-\mathrm{Ct}_{\mathrm{g}}$, where $\Delta \mathrm{Ct}$ is the normalized value, $\mathrm{Ct}_{\mathrm{p}}$ is the $\mathrm{Ct}$ obtained from amplification of the phytoplasmatic $16 \mathrm{~S} r R N A$ gene, and $\mathrm{Ct}_{\mathrm{g}}$ is the $\mathrm{Ct}$ obtained from amplification of the grapevine chaperonin gene, the endogenous control used in the reaction. $\Delta \mathrm{Ct}$ values were compared through oneway analysis of variance, followed by Tukey's Exact Test, performed in the SPSS statistical package for Windows (v. 24.0; IBM Corporation) to determine whether the symptom severity of the disease was correlated to different phytoplasma relative abundance in the plants. Correlation coefficient $\left(R^{2}\right)$ was calculated by linear regression model.

BNp characterization by multiple gene sequence analyses. ' $\mathrm{Ca}$. P. solani' strains, detected in grapevine plants, were characterized by nucleotide sequence analyses of the genes tufB, vmpl, and stamp.

Identification of tufB types, commonly present in Italy (tufB-a and tufB-b) (Mori et al. 2015), was performed using the TaqMan allelic discrimination assay, employing tufB type-specific probes carrying different fluorescent dyes, according to Berger et al. (2009).

The vmpl gene was amplified in an automated thermal cycler C1000 Cycler Touch (Bio-Rad) using the StolH10F1/StolH10R1 primer pair (Cimerman et al. 2009), followed by nested PCR with the TYPH10F/TYPH10R primer pair, using mixtures and PCR conditions as described by Fialová et al. (2009). Nested PCR amplicons were verified through electrophoresis on $1 \%$ agarose gels in Tris-borate-EDTA (TBE) buffer. Then, RFLP analysis was performed using the RsaI restriction enzyme (Pacifico et al. 2009), according to the manufacturer's instructions (New England BioLabs). Digestion fragments were separated through electrophoresis on $3 \%$ agarose gels in TBE buffer stained with Gel-Red (Biotum) and visualized under UV transilluminator. Attribution of BNp strains identified in the present study to vmpl RFLP types (V types) was determined by comparison of their RsaI-RFLP patterns with vmpl digestion profiles previously described in accordance with SEE-ERANET nomenclature (Foissac et al. 2013; Quaglino et al. 2016). The $v m p l$ amplicons, representative of the identified $\mathrm{V}$ types, were sequenced ( $5 \times$ coverage per base position) by a commercial service (Eurofins Genomics). Nucleotide sequences were assembled by the Contig Assembling Program and trimmed to the annealing sites of the primers TYPH10F/TYPH10R in the software BioEdit, version 7.2.6 (Hall 1999). To confirm the attribution to $\mathrm{V}$ types, trimmed nucleotide sequences were searched for single-nucleotide polymorphisms (SNP) in recognition sites of the enzyme RsaI through virtual RFLP analyses using the software pDRAW32 (http://www.acaclone.com/). Moreover, vmpl nucleotide sequences were aligned using ClustalW Multiple Alignment and analyzed by Sequence Identity Matrix in the software BioEdit. Attribution to vmpl sequence variants was carried out by comparison with sequences previously deposited in the GenBank database. In detail, nucleotide sequences of the same variant shared $100 \%$ sequence identity.

The stamp gene was amplified in an automated thermal cycler C1000 Cycler Touch using StampF/StampR0 and StampF1/StampR1 primer pairs in direct and nested PCR, respectively, following PCR conditions as described by Fabre et al. (2011). Nested PCR amplicons were verified through electrophoresis on $1 \%$ agarose gels in TBE buffer. All obtained stamp amplicons were sequenced and analyzed as described for gene vmpl. 
To further characterize the vmpl and stamp gene sequence variants, their nucleotide sequences were translated in silico and searched for the presence of nonsynonymous and synonymous SNP, and other sequence modifications (insertions or deletions).

Collective vmp1/stamp types were determined combining the vmpl and stamp sequence variant of each BNp strain identified in grapevine, as previously described (Quaglino et al. 2016).

Phylogenetic analysis, association of BNp strains with symptom severity, and selective pressure on BNp strain population. Representative nucleotide sequences of $v m p 1$ and stamp sequence variants, identified in this and previous studies (Quaglino et al. 2016), were utilized for phylogenetic analyses. Moreover, representative nucleotide sequences of vmpl and stamp genes were concatenated by BioEdit and employed for phylogenetic analyses. In detail, unrooted phylogenetic trees were generated by minimum evolution method carried out using the Jukes-Cantor model and bootstrap replicated 1,000 times in the MEGA7 software (Tamura et al. 2013).

The association between BNp strains (identified in 2016 and grouped in distinct vmpl, stamp, and vmpl/stamp phylogenetic clusters) and BN symptom severity was determined as the difference in their distribution in grapevines showing symptom severity class 1 , 2 , and 3 in 2016 through statistical analyses using a $\chi^{2}$ test in SPSS. Due to the analyzed sample size and to the low number of variables (same vineyard, clonal grapevine cultivar, sampling time, molecular analysis, and operator), the $P$ value significance was set at $<0.1$, the upper limit of significance to exclude the random effect (Fisher 1950), as reported in previous studies (Quaglino et al. 2016; Raudales et al. 2009; von Diest et al. 2016).

Moreover, distinct vmpl, stamp, and vmpl/stamp phylogenetic clusters, including BNp strains identified in 2016, were ranked in accordance with (i) their average disease severity $(\mathrm{A} S)$, calculated using the formula $\mathrm{A} S=\Sigma\left(c \cdot f^{\mathrm{A} S}\right) / n^{\mathrm{A} S}$, where $\mathrm{A} S$ is the average disease severity of the cluster, $c$ is the symptom severity class, $f^{\mathrm{A} S}$ is the frequency of the symptom severity class in grapevines infected by $\mathrm{BNp}$ strains of the cluster, and $n^{\mathrm{A} S}$ is the number of symptomatic grapevines infected by $\mathrm{BNp}$ strains of the cluster; and (ii) their $\mathrm{OS}$ percentage $(\mathrm{OS} \%)$, calculated using the formula OS\% $=\left[\Sigma\left(c \cdot f^{\mathrm{A} S}\right) / n\right]$. $100 / S$, where OS\% describes how much each cluster contributes to the disease severity in the vineyard, $c$ is the symptom severity class, $f^{\mathrm{A} S}$ is the frequency of the symptom severity class in grapevines infected by BNp strains of the cluster, $n$ is the total number of symptomatic grapevines, and $S$ is the disease severity (as described above).

A codon-based $Z$ test of positive selection was performed using the Nei-Gojobori method with MEGA7 to determine the $d N / d S$ ratio and to calculate the probability of rejecting the null hypothesis of strict neutrality $(d N=d S)$ in favor of the positive selection hypothesis $(d N>d S)$. $d S$ and $d N$ are the numbers of synonymous and nonsynonymous substitutions per site, respectively. The variance of the difference was computed using the bootstrap method (1,000 replicates). Analyses were conducted according to Nielsen (2005) and Murolo and Romanazzi (2016). The overall $d N / d S$ ratio > 1.0 and $P$ value $<0.05$ means positive selection, while ratio $=1$ or $<$ 1.0 means neutral or purifying selection process, respectively (Murolo et al. 2014; Nei and Kumar 2000). The analysis involved 40 and 66 nucleotide sequences of the genes vmpl and stamp, respectively. All positions with less than $95 \%$ site coverage were eliminated. That is, fewer than 5\% alignment gaps, missing data, and ambiguous bases were allowed at any position. There were 517 and 158 codon positions in the final dataset for the genes vmpl and stamp, respectively.

\section{RESULTS}

Symptom observations. In 2015, 24 of 735 (3.3\%) grapevine plants showed typical GY symptoms. Symptom severity classes were observed in the vineyard and the most represented was the symptom severity class 2 (37.5\% of the symptomatic vines), followed by symptom severity class $3(33.3 \%)$ and class $1(29.2 \%)$ (Table 1).

In 2016, 53 of 734 (7.2\%) grapevine plants showed typical GY symptoms: (i) 33 of 53 plants exhibited GY symptoms for the first time, (ii) 20 of 53 plants showed symptoms in both years, (iii) 3 plants showing symptoms in 2015 were symptomless in 2016, and (iv) 1 plant showing symptoms in 2015 was eradicated after death. As in 2015, all three symptom severity classes were observed in the vineyard and the most represented was symptom severity class 3 (47.2\% of the symptomatic vines), followed by symptom severity class 1 and 2, which were equally abundant (26.4\% each). OS was determined as 2.04 in 2015 and 2.20 in 2016.

GY phytoplasma detection and relative quantification. Real-time PCR, carried out by TaqMan assays performed using primer pairs specific for the amplification of BNp and FDp 16S rDNA, detected BNp in every symptomatic grapevine plant in 2015 whereas, in 2016, it was detected in 45 of 53 symptomatic grapevine plants. In both years, FDp was never detected in symptomatic plants. BNp and FDp were never detected in symptomless plants, including the three plants that no longer showed symptoms in 2016. In both years, $\mathrm{Ct}$ values obtained by amplification of BNp 16S rDNA varied among samples $(27<\mathrm{Ct}<34)$, while those obtained for chaperonin were slightly less variable $(16<\mathrm{Ct}<20) ; \Delta \mathrm{Ct}$ values varied from 2 to 11 (Tables 1 and 2). Assay reliability was confirmed by the results obtained from the controls: (i) healthy control plants and reaction mixtures devoid of DNA gave no amplification and (ii) IC gave expected amplification for $\mathrm{BNp}$ (average $\mathrm{Ct} 31$ ) and FDp subgroup $16 \mathrm{SrV}-\mathrm{C}$ (average $\mathrm{Ct} 28$ ) and $16 \mathrm{SrV}-\mathrm{D}$ (average $\mathrm{Ct} 28$ ). No statistically significant differences $\left(R^{2}=0.008, P=0.57\right)$ between symptom severity of grapevine and $\Delta \mathrm{Ct}$ values were obtained.

BNp strain characterization by multiple gene typing. tufB gene molecular characterization was carried out on BNpinfected grapevine plants (24 in 2015 and 45 in 2016). TaqMan allelic discrimination assays revealed that $\mathrm{BNp}$ strains that infected symptomatic grapevines in both years are classified as tufB type $b$.

Of $69 \mathrm{BNp}$ strains identified in 24 and 45 symptomatic vines in 2015 and 2016, respectively, 57 (15 of 24 from 2015 and 42 of 45 from 2016) yielded vmpl nested-PCR amplicons (TYPH10F/ TYPH10R) that were typed through digestion using the RsaI enzyme. BNp strains identified in 2015 showed the presence of two $R s a I-R F L P$ profiles attributed to vmpl types V11 (six strains) and V12 (nine strains). BNp strains identified in 2016 showed the presence of three actual RsaI-RFLP profiles attributed to $v m p 1$ types V11 (24 strains), V12 (15 strains), and V9 (3 strains). Comprehensive attribution to $\mathrm{V}$ type was confirmed by in silico RFLP analysis (Fig. 1). Ten BNp strains, identified in the same symptomatic plants in both years, showed undistinguishable RsaI-RFLP patterns.

The vmpl sequence analysis was carried out on $40 \mathrm{BNp}$ strains $(9$ from 2015 and 31 from 2016), representative of the $V$ types identified by RFLP digestions (Tables 1 and 2). Based on sequence identities, 12 vmpl sequence variants (here named vmFi1 to vmFi12) have been identified within BNp strain populations. Sequence variants vmFi1 (prevalent in both years), vmFi2, and vmFi3 were present in both years; vmFi4 was detected only in 2015 and vmFi5 to vm12 only in 2016 (Tables 1 and 2). Comparison with the vmpl sequence variant dataset updated in the present study (Supplementary Table S1) revealed that vmFi1, vmFi2, vmFi3, vmFi5, and vmFi6 shared $100 \%$ sequence identity with previously reported sequence variants $\mathrm{Vm} 43$, Vm45, Vm42, Vm39, and Vm41, respectively. Other vmpl sequence variants (vmFi4 and vmFi7 to vmFi12) reported in the present study were not identical to any other vmpl sequences in the dataset but shared the best identities versus sequence variants Vm39, Vm41, $\mathrm{Vm} 42, \mathrm{Vm} 43$, and Vm45. Further analyses carried out on nucleotide and in silico translated $\mathrm{vmpl}$ gene sequences showed that the genetic diversity among sequence variants vmFi 1 to vmFi12 is caused by the presence of 125 SNP (116 nonsynonymous and 9 synonymous) and five insertions determining different lengths of the gene vmpl and the 
coded Vmp1 protein (Supplementary Fig. S1; Supplementary Table S2). New vmpl sequence variants shared high nucleotide sequence identity ( 99.4 to $99.9 \%$ ) but were distinguished from closely related sequence variants previously published (Vm39, Vm41, Vm42, Vm43, and Vm45) by nonsynonymous SNP (Supplementary Table $\mathrm{S} 3$ ). For each vmpl sequence variant, one representative nucleotide sequence was deposited to the National Center for Biotechnology Information (NCBI) GenBank at the accession number shown in Table 3 (vmFil to vmFi12).

Of $69 \mathrm{BNp}$ strains identified in 24 and 45 symptomatic vines in 2015 and 2016, respectively, 66 (22 from 2015 and 44 from 2016) yielded stamp nested-PCR amplicons (StampF1/StampR1) of the expected size (approximately $500 \mathrm{bp}$ ) that were typed by nucleotide sequence analysis. Based on sequence identities, 16 stamp sequence variants (here named stFi1 to stFi16) have been identified within BNp strain populations. Sequence variants stFi1 (prevalent in 2016), stFi2 (prevalent in 2015), stFi3, and stFi4 were present in both years; stFi5 to stFi9 were detected only in 2015 and stFi10 to stFi16 only in 2016 (Tables 1 and 2). Comparison with the stamp sequence variant dataset updated in the present study (Supplementary Table S4) revealed that stFi1, stFi2, stFi3, and stFi4 shared $100 \%$ sequence identity with previously reported sequence variants St10, St5, St18, and St22, respectively. Other stamp sequence variants (stFi5 to stFi16) reported in the present study are not identical to any other stamp sequences in the dataset but shared the best identities versus sequence variants St5, St10, St15, St18, St22, St36, and St37. Further analyses carried out on nucleotide and in silico translated stamp gene sequences showed that the genetic diversity among sequence variants stFi1 to stFi16 is caused by the presence of 50 SNP (42 nonsynonymous and 8 synonymous) and one insertion (nucleotides 253 to 256) (Supplementary Fig. S2). New stamp sequence variants shared high nucleotide sequence identity ( 95.9 to $99.7 \%$ ) but were distinguished from closely related sequence variants previously published (St5, St10, St15, St18, St22, St36, and St37) by nonsynonymous SNP (Supplementary Table S5). For each stamp sequence variant, one representative nucleotide sequence was deposited to NCBI GenBank at accession numbers shown in Table 4 (stFi1 to stFi16).
TABLE 2. Symptom severity class (Sev), relative abundance, and strain type of bois noir phytoplasma identified in symptomatic grapevine plants in Greve in Chianti vineyard in 2016

\begin{tabular}{|c|c|c|c|c|c|}
\hline \multirow[b]{3}{*}{ Plant } & \multicolumn{5}{|c|}{2016} \\
\hline & \multirow[b]{2}{*}{$\mathrm{Sev}$} & \multirow[b]{2}{*}{$\Delta \mathrm{Ct}$} & \multirow[b]{2}{*}{ RFLP } & \multicolumn{2}{|c|}{ Sequence variant } \\
\hline & & & & vmpl & stamp \\
\hline $\operatorname{San} 25$ & 3 & 5.6 & V11 & vmFi1 & stFi1 \\
\hline San26 & 1 & $\ldots$ & $\ldots$ & $\ldots$ & $\ldots$ \\
\hline San27 & 1 & $\ldots$ & $\ldots$ & $\ldots$ & $\ldots$ \\
\hline $\operatorname{San} 28$ & 2 & 5.58 & V11 & vmFil & stFi14 \\
\hline $\operatorname{San} 29$ & 3 & 4.88 & - & - & stFi15 \\
\hline $\operatorname{San} 30$ & 2 & $\ldots$ & $\ldots$ & $\ldots$ & $\ldots$ \\
\hline San31 & 3 & 8.02 & V9 & vmFi5 & stFi2 \\
\hline San32 & 1 & $\ldots$ & V12 & vmFi12 & stFi3 \\
\hline San33 & 3 & 4.9 & V11 & $\ldots$ & stFi2 \\
\hline San34 & 3 & 10.34 & V11 & vmFil & stFi1 \\
\hline San35 & 1 & & V12 & vmFi12 & stFi2 \\
\hline San36 & 2 & 6.9 & V9 & vmFi5 & stFi2 \\
\hline San37 & 3 & 7.13 & V9 & vmFi9 & stFi2 \\
\hline San38 & 1 & 6.08 & V11 & $\ldots$ & stFi1 \\
\hline San39 & 3 & 7.1 & V12 & $\ldots$ & stFi3 \\
\hline San40 & 3 & 6.09 & V11 & vmFil & stFi1 \\
\hline San41 & 1 & 10.06 & $\ldots$ & $\ldots$ & $\ldots$ \\
\hline $\operatorname{San} 42$ & 2 & 7.59 & V11 & vmFi1 & stFi1 \\
\hline $\operatorname{San} 43$ & 3 & 4.79 & V12 & vmFi12 & stFi3 \\
\hline San44 & 1 & & & $\ldots$ & \\
\hline $\operatorname{San} 45$ & 2 & 5.47 & $\ldots$ & $\ldots$ & stFi16 \\
\hline San46 & 3 & 5.16 & V11 & vmFi1 & stFi1 \\
\hline San47 & 2 & 4.97 & V11 & vmFi6 & stFi2 \\
\hline $\operatorname{San} 48$ & 1 & $\ldots$ & $\ldots$ & $\ldots$ & $\ldots$ \\
\hline San49 & 3 & 8.61 & V12 & vmFi7 & stFi3 \\
\hline San50 & 2 & 6.88 & V11 & vmFi1 & stFi1 \\
\hline San51 & 3 & 5.82 & V11 & vmFil & stFi1 \\
\hline San52 & 2 & 5.68 & V11 & vmFi6 & stFi2 \\
\hline San53 & 2 & 8.79 & V11 & vmFi1 & stFi1 \\
\hline San54 & 3 & 2.59 & V12 & vmFi7 & stFi3 \\
\hline San55 & 3 & $\ldots$ & V12 & vmFi3 & $\mathrm{stFi} 2$ \\
\hline San56 & 3 & 6.42 & V11 & vmFi11 & stFi1 \\
\hline San57 & 1 & 4.56 & V11 & vmFi1 & stFi1 \\
\hline
\end{tabular}

a Abbreviations: $\Delta \mathrm{Ct}=$ relative abundance of bois noir phytoplasma and RFLP $=v m p 1$ restriction fragment length polymorphism type.

TABLE 1. Symptom severity class (Sev), relative abundance, and strain type of bois noir phytoplasma identified in symptomatic grapevine plants in Greve in Chianti vineyard over 2015 to $2016^{\mathrm{a}}$

\begin{tabular}{|c|c|c|c|c|c|c|c|c|c|c|}
\hline \multirow[b]{3}{*}{ Plant } & \multicolumn{5}{|c|}{2015} & \multicolumn{5}{|c|}{2016} \\
\hline & \multirow[b]{2}{*}{$\mathrm{Sev}$} & \multirow[b]{2}{*}{$\Delta \mathrm{Ct}$} & \multirow[b]{2}{*}{ RFLP } & \multicolumn{2}{|c|}{ Sequence variant } & \multirow[b]{2}{*}{$\mathrm{Sev}$} & \multirow[b]{2}{*}{$\Delta \mathrm{Ct}$} & \multirow[b]{2}{*}{ RFLP } & \multicolumn{2}{|c|}{ Sequence variant } \\
\hline & & & & $v m p 1$ & stamp & & & & vmpl & stamp \\
\hline San 1 & 3 & 7 & $\ldots$ & $\ldots$ & $\ldots$ & 3 & 6.08 & V11 & $\ldots$ & $\ldots$ \\
\hline San2 & 3 & 7.5 & V12 & vmFi2 & stFi3 & 3 & 6.54 & V12 & vmFi2 & stFi3 \\
\hline San3 & 1 & 4.5 & $\ldots$ & $\ldots$ & stFi5 & 0 & $\ldots$ & $\ldots$ & $\ldots$ & $\ldots$ \\
\hline San4 & 1 & 10 & $\ldots$ & $\ldots$ & stFi6 & 2 & 6.95 & V11 & $\ldots$ & stFi1 \\
\hline San5 & 2 & 6.5 & V11 & vmFi1 & stFi1 & 3 & 6.69 & V11 & vmFi10 & stFi1 \\
\hline San6 & 2 & 6.5 & $\ldots$ & $\ldots$ & stFi7 & 2 & 7.53 & V12 & - & stFi10 \\
\hline $\operatorname{San} 7$ & 1 & 9 & V11 & vmFi1 & stFi1 & 1 & 5.83 & V11 & vmFi1 & stFi1 \\
\hline San8 & 3 & 5.5 & $\ldots$ & $\ldots$ & stFi2 & 3 & 7.17 & V12 & $\ldots$ & stFi11 \\
\hline San9 & 3 & 8 & V12 & $\ldots$ & stFi2 & Erad & $\ldots$ & $\ldots$ & $\ldots$ & $\ldots$ \\
\hline San 10 & 2 & 8.5 & $\ldots$ & $\ldots$ & stFi8 & 3 & 7.38 & V12 & $\ldots$ & $\mathrm{stFi} 2$ \\
\hline San11 & 2 & 7 & V12 & vmFi2 & stFi3 & 3 & 4.97 & V12 & vmFi8 & stFi3 \\
\hline San12 & 1 & 6 & V12 & $\ldots$ & stFi2 & 0 & $\ldots$ & $\ldots$ & $\ldots$ & $\ldots$ \\
\hline San13 & 1 & 10.5 & V12 & $\ldots$ & stFi2 & 1 & 2.12 & $\ldots$ & $\ldots$ & $\ldots$ \\
\hline San14 & 3 & 7 & $\ldots$ & $\ldots$ & stFi2 & 2 & 8.07 & V12 & $\ldots$ & stFi2 \\
\hline San 15 & 3 & 8.5 & V11 & $\ldots$ & stFi1 & 1 & 10.41 & V11 & $\ldots$ & $\ldots$ \\
\hline San 16 & 3 & 7 & V11 & vmFi1 & stFi1 & 3 & 6.27 & V11 & vmFi1 & stFi12 \\
\hline San17 & 2 & 8 & V11 & vmFi1 & stFi1 & 1 & 7.68 & V11 & vmFil & stFi13 \\
\hline San 18 & 1 & 11 & V12 & $\ldots$ & stFi3 & 0 & $\ldots$ & $\ldots$ & $\ldots$ & $\ldots$ \\
\hline San 19 & 1 & 8 & V12 & $\ldots$ & stFi2 & 2 & 1.04 & $\ldots$ & $\ldots$ & $\mathrm{stFi} 2$ \\
\hline $\operatorname{San} 20$ & 2 & 6 & $\ldots$ & $\ldots$ & $\ldots$ & 3 & 7.66 & $\ldots$ & $\ldots$ & stFi3 \\
\hline $\operatorname{San} 21$ & 3 & 6.5 & V11 & vmFi1 & stFi1 & 3 & 7.18 & V11 & vmFi1 & stFil \\
\hline San22 & 2 & 6.5 & $\ldots$ & $\ldots$ & stFi9 & 1 & 6.73 & V11 & $\ldots$ & stFi1 \\
\hline San23 & 2 & 7 & V12 & vmFi3 & stFi2 & 3 & 5.01 & V12 & vmFi3 & $\mathrm{stFi} 2$ \\
\hline $\operatorname{San} 24$ & 2 & 6.5 & V12 & vmFi4 & stFi4 & 2 & 5.64 & V12 & vmFi3 & stFi4 \\
\hline
\end{tabular}

a Abbreviations: $\Delta \mathrm{Ct}=$ relative abundance of bois noir phytoplasma, $\mathrm{RFLP}=v m p 1$ restriction fragment length polymorphism type, and Erad $=$ eradicated. 
Through the combination of $v m p l$ and stamp nucleotide sequences available for $40 \mathrm{BNp}$ strains over the 2 years, $17 \mathrm{vmpl} / \mathrm{stamp}$ types were identified. The type vmFi1/stFi1 (identical to Vm43/St10) represents the most widespread type combination for both years: $55.5 \%$ in 2015 and $35.3 \%$ in 2016.

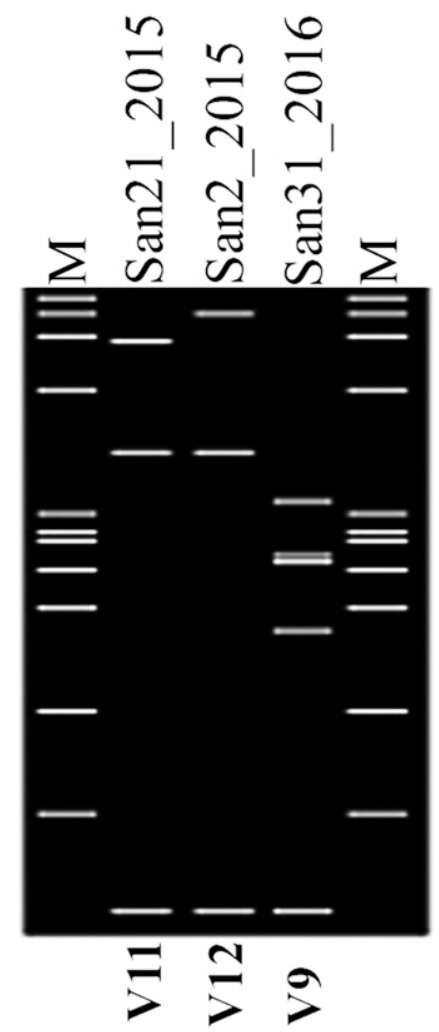

Fig. 1. Virtual $R s a$ I-restriction fragment length polymorphism (RFLP) profiles of gene vmpl obtained from bois noir phytoplasma (BNp) strains detected in the study vineyard in 2015 and 2016. Virtual RsaI restriction profiles of vmp1 were obtained by digesting trimmed TYPH10F/TYPH10R fragments with the software pDRAW32. San21_2015 represents 30 BNp strains exhibiting the RsaI-RFLP profile of V11, San2_2015 represents 24 BNp strains exhibiting the RsaI-RFLP profile of V12, San31_2016 represents 3 BNp strains exhibiting the RsaI-RFLP profile of V9, and $\mathrm{M}=$ marker $\Phi 174 \mathrm{DNA} / \mathrm{Hae} \mathrm{III}$ (Promega Corp.). Fragment length from top to bottom: 1,353, 1,078, 872, 603, $310,281,271,234,194,118$, and 72 bp.

TABLE 3. Genetic variants of $v m p 1$ identified among bois noir phytoplasma strains identified in the vineyard in Greve in Chianti over 2015 to 2016: prevalence, representative strains, and sequence accession numbers

\begin{tabular}{|c|c|c|c|c|}
\hline \multirow[b]{2}{*}{ Variant } & \multicolumn{2}{|c|}{$\begin{array}{l}\text { Number of } \\
\text { strains }\end{array}$} & \multirow[b]{2}{*}{ Representative strain ${ }^{\mathrm{a}}$} & \multirow[b]{2}{*}{ Accession ${ }^{\mathrm{b}}$} \\
\hline & 2015 & 2016 & & \\
\hline vmFil (Vm43) & 5 & 14 & San21_2015 & MF182856 \\
\hline vmFi2 (Vm45) & 2 & 1 & San2_2015 & MF182857 \\
\hline vmFi3 (Vm42) & 1 & 3 & San24_2016 & MF182861 \\
\hline vmFi4 & 1 & 0 & San24_2015 & MF182858 \\
\hline vmFi5 (Vm39) & 0 & 2 & San31_2016 & MF182859 \\
\hline vmFi6 (Vm41) & 0 & 2 & San47_2016 & MF182860 \\
\hline vmFi7 & 0 & 2 & San49_2016 & MF182862 \\
\hline vmFi8 & 0 & 1 & San11_2016 & MF182863 \\
\hline vmFi9 & 0 & 1 & San37_2016 & MF182864 \\
\hline vmFi10 & 0 & 1 & San5_2016 & MF182865 \\
\hline vmFi11 & 0 & 1 & San56_2016 & MF182866 \\
\hline vmFi12 & 0 & 3 & San43_2016 & MF182867 \\
\hline
\end{tabular}

a The name of each representative strain is composed of plant name and year of sampling, separated by an underscore.

${ }^{b}$ Accession number linked to sequences deposited to the National Center for Biotechnology Information GenBank.
Phylogenetic analysis, association of $\mathrm{BNp}$ strains with symptom severity, and selective pressure on $\mathrm{BNp}$ strain population. A phylogenetic tree, generated employing representative $v m p 1$ sequence variants from the dataset ( $\mathrm{Vm} 1$ to $\mathrm{Vm} 80)$ and from the present study (vmFi1 to vmFi12), identified the presence of four main clusters. Sequence variants, here identified in symptomatic grapevines, grouped into clusters 1 (vmFi2, vmFi3, vmFi4, vmFi6, vmFi7, vmFi8, and vmFi12), 2 (vmFi5 and vmFi9), and 3 (vmFi1, vmFi10, and vmFi11) (Fig. 2A). Statistically significant differences were observed in the distribution of $\mathrm{BNp}$ strains belonging to vmpl phylogenetic cluster 3 (AS 2.37, OS\% 32.5) in grapevine plants showing symptom severity class 1,2 , and $3\left(\chi^{2}=4.667, P=0.097\right)$. No statistically significant differences were observed in the distribution of BNp strains belonging to $v m p 1$ phylogenetic clusters 1 (AS 2.42, OS\% 24.8) and 2 (AS 2.67, OS\% 6.8) (Fig. 2B).

A phylogenetic tree generated by employing representative stamp sequence variants from the dataset (St1 to St46) and from the present study (stFi1 to stFi16) identified the presence of three main clusters. Sequence variants, here identified in symptomatic grapevines, grouped into clusters 1 (stFi3, stFi6, stFi8, stFi10, and stFi16), 2 (stFi2, stFi4, stFi11, and stFi15), and 3 (stFi1, stFi5, stFi9, stFi12, stFi13, and stFi14); sequence variant stFi7 did not group in identified clusters (Fig. 3A). Statistically significant differences were observed in the distribution of BNp strains belonging to stamp phylogenetic cluster 1 (AS 2.60, OS\% 22.2) in grapevine plants showing symptom severity class 1,2 , and $3\left(\chi^{2}=8, P=0.018\right)$. No statistically significant differences were observed in the distribution of BNp strains belonging to stamp phylogenetic clusters 2 (AS 2.47, OS\% 31.6) and 3 (AS 2.21, OS\% 35.9) (Fig. 3B).

A phylogenetic tree generated by employing concatenated $v m p 1$ and stamp gene nucleotide sequences representative of $66 \mathrm{vmpl/}$ stamp types (49 from the dataset [Supplementary Table S6] and 17 from the present study] identified the presence of eight main clusters. vmpl/stamp types, here identified in symptomatic grapevines, grouped into clusters 1 (vmFi1/stFi1, vmFi1/stFi12, vmFi1/stFi13, vmFi1/ stFi14, vmFi10/stFi1, and vmFi11/stFi1), 3 (vmFi2/stFi3, vmFi8/ stFi3, vmFi12/stFi2, and vmFi12/stFi3), 4 (vmFi3/stFi2, vmFi3/stFi4, vmFi4/stFi4, vmFi6/stFi2, and vmFi7/stFi3), and 5 (vmFi5/stFi2 and vmFi9/stFi2) (Fig. 4A). Further analyses of the nucleotide sequence alignment of vmpl/stamp types identified in the Chianti Classico area revealed that $\mathrm{BNp}$ strains within each cluster (intracluster heterogeneity) shared a mean sequence identity of $99.57 \%$ (approximately 10

TABLE 4. Genetic variants of stamp identified among bois noir phytoplasma strains identified in the vineyard in Greve in Chianti over 2015 to 2016: prevalence, representative strains, and sequence accession numbers

\begin{tabular}{|c|c|c|c|c|}
\hline \multirow[b]{2}{*}{ Variant } & \multicolumn{2}{|c|}{$\begin{array}{c}\text { Number of } \\
\text { strains }\end{array}$} & \multirow[b]{2}{*}{ Representative strain $^{\mathrm{a}}$} & \multirow[b]{2}{*}{ Accession $^{\mathrm{b}}$} \\
\hline & 2015 & 2016 & & \\
\hline stFi1 (St10) & 5 & 16 & San21_2015 & MF182868 \\
\hline stFi2 (St5) & 7 & 12 & San23_2015 & MF182869 \\
\hline stFi3 (St18) & 3 & 8 & San2_2015 & MF182870 \\
\hline stFi4 (St22) & 1 & 1 & San24_2015 & MF182871 \\
\hline stFi5 & 1 & 0 & San3_2015 & MF182872 \\
\hline stFi6 & 1 & 0 & San4_2015 & MF182873 \\
\hline stFi7 & 1 & 0 & San6_2015 & MF182874 \\
\hline stFi8 & 1 & 0 & San10_2015 & MF182875 \\
\hline stFi9 & 1 & 0 & San22_2015 & MF182876 \\
\hline stFi10 & 0 & 1 & San6_2016 & MF182877 \\
\hline stFi11 & 0 & 1 & San8_2016 & MF182878 \\
\hline stFi12 & 0 & 1 & San16_2016 & MF182879 \\
\hline stFi13 & 0 & 1 & San17_2016 & MF182880 \\
\hline stFi14 & 0 & 1 & San28_2016 & MF182881 \\
\hline stFi15 & 0 & 1 & San29_2016 & MF182882 \\
\hline stFi16 & 0 & 1 & San45_2016 & MF182883 \\
\hline
\end{tabular}

a The name of each representative strain is composed of plant name and year of sampling, separated by an underscore.

b Accession number linked to sequences deposited to the National Center for Biotechnology Information GenBank. 


\section{A}

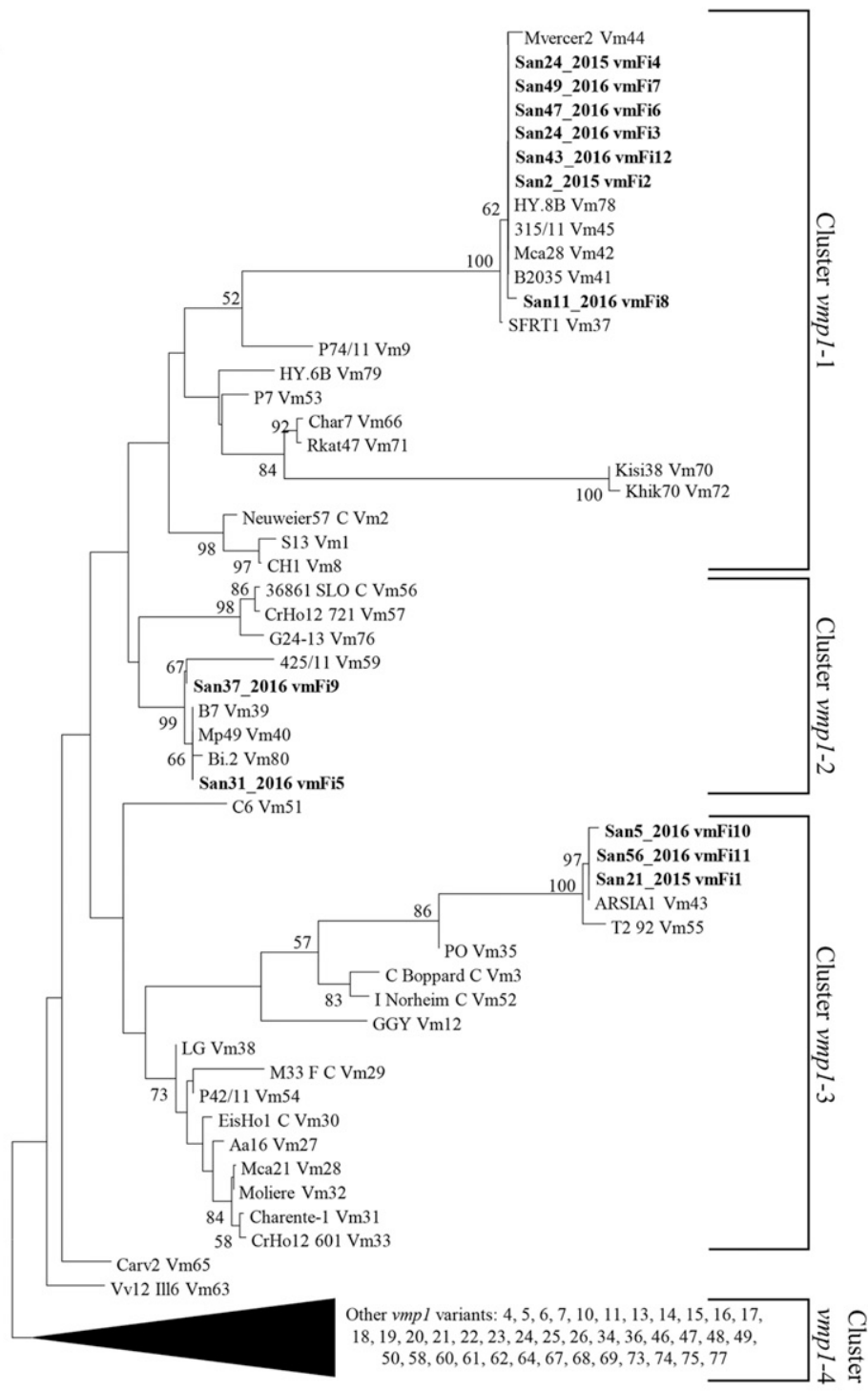

$\stackrel{\longmapsto}{\longrightarrow 0050}$

B

Cluster

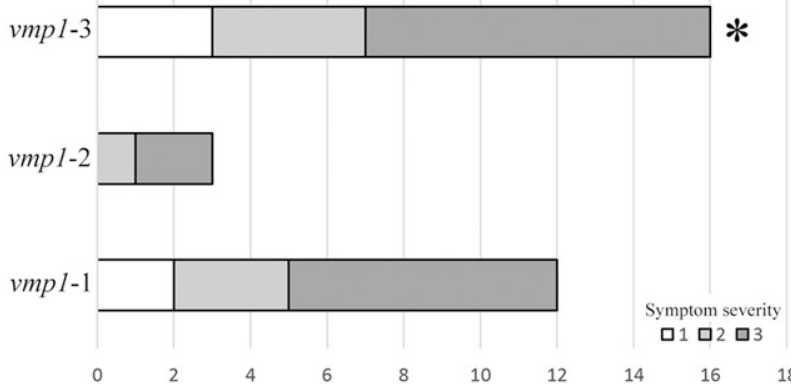

$\mathrm{A} S \quad \mathrm{OS} \%$

$2.37 \quad 32.5$

$2.67 \quad 6.8$

$2.42 \quad 24.8$

Number of Sangiovese plants infected by BNp in the study vineyard

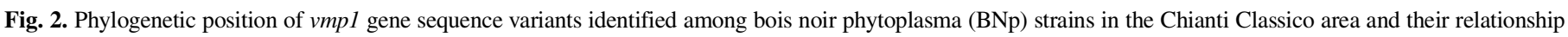

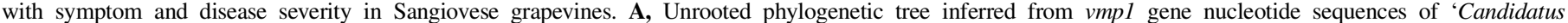

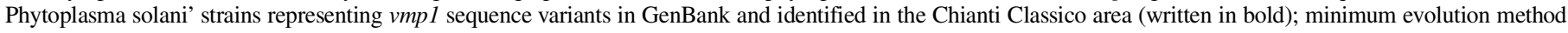

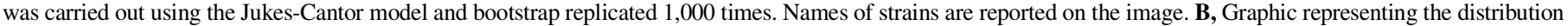

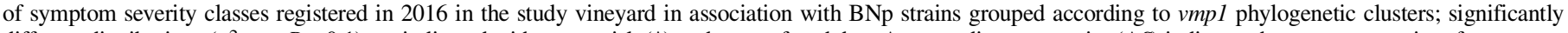

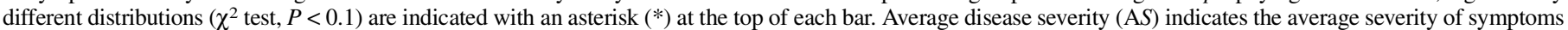

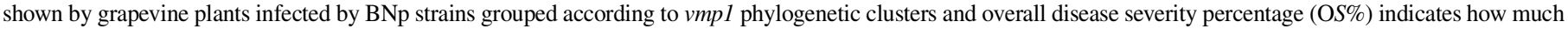
each BNp strain cluster contributes to the severity of the disease observed in the study vineyard in 2016. 

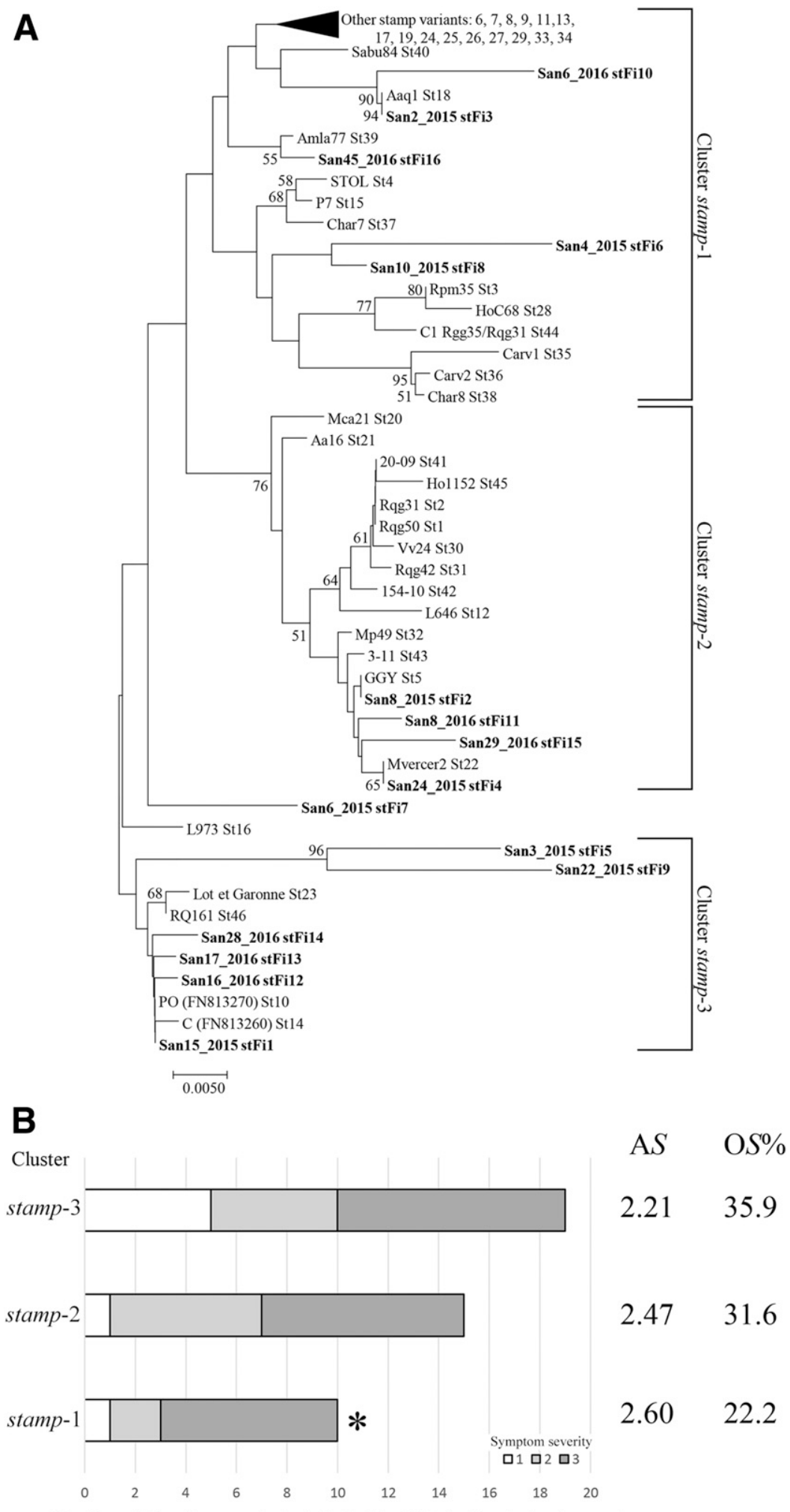

Fig. 3. Phylogenetic position of stamp gene sequence variants identified among bois noir phytoplasma (BNp) strains in the Chianti Classico area and their relationship with symptom and disease severity in Sangiovese grapevines. A, Unrooted phylogenetic tree inferred from stamp gene nucleotide sequences of 'Candidatus Phytoplasma solani' strains representing stamp sequence variants in GenBank and identified in the Chianti Classico area (written in bold); minimum evolution method was carried out using the Jukes-Cantor model and bootstrap replicated 1,000 times. Names of strains are reported on the image. B, Graphic representing the distribution of symptom severity classes registered in 2016 in the study vineyard in association with BNp strains grouped according to stamp phylogenetic clusters; significantly different distributions $\left(\chi^{2}\right.$ test, $\left.P<0.1\right)$ are indicated with an asterisk $(*)$ at the top of each bar. Average disease severity $($ A $S)$ indicates the average severity of symptoms shown by grapevine plants infected by BNp strains grouped according to stamp phylogenetic clusters and overall disease severity percentage (OS\%) indicates how much each BNp strains cluster contributes to the severity of the disease observed in the vineyard in 2016. 
SNP distinguishing one strain from another), whereas BNp strains of distinct clusters (intercluster heterogeneity) shared a mean sequence identity of $89.41 \%$ (approximately 184 SNP distinguishing one strain from another) (Table 5). Statistically significant differences were observed in the distribution of BNp strains belonging to vmpl/stamp phylogenetic cluster $1\left(\chi^{2}=4.667, P=0.097, \mathrm{AS} 2.37\right.$, OS\% 32.5) and $3\left(\chi^{2}=7, P=0.030, \mathrm{~A} S 2.67, \mathrm{O} \%\right.$ 13.7) in grapevine plants showing symptom severity class 1,2 , and 3 . Instead, no statistically significant

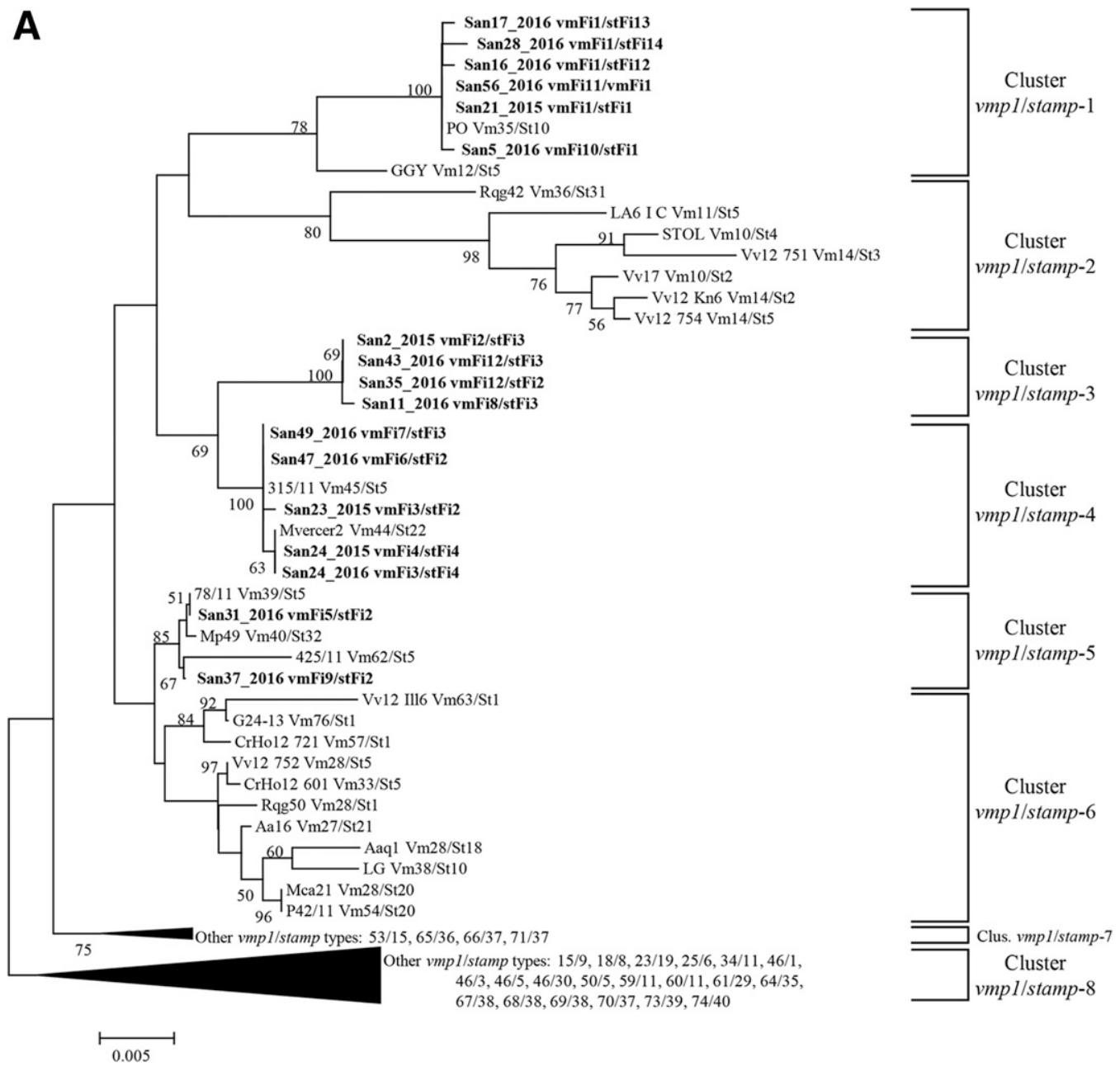

B

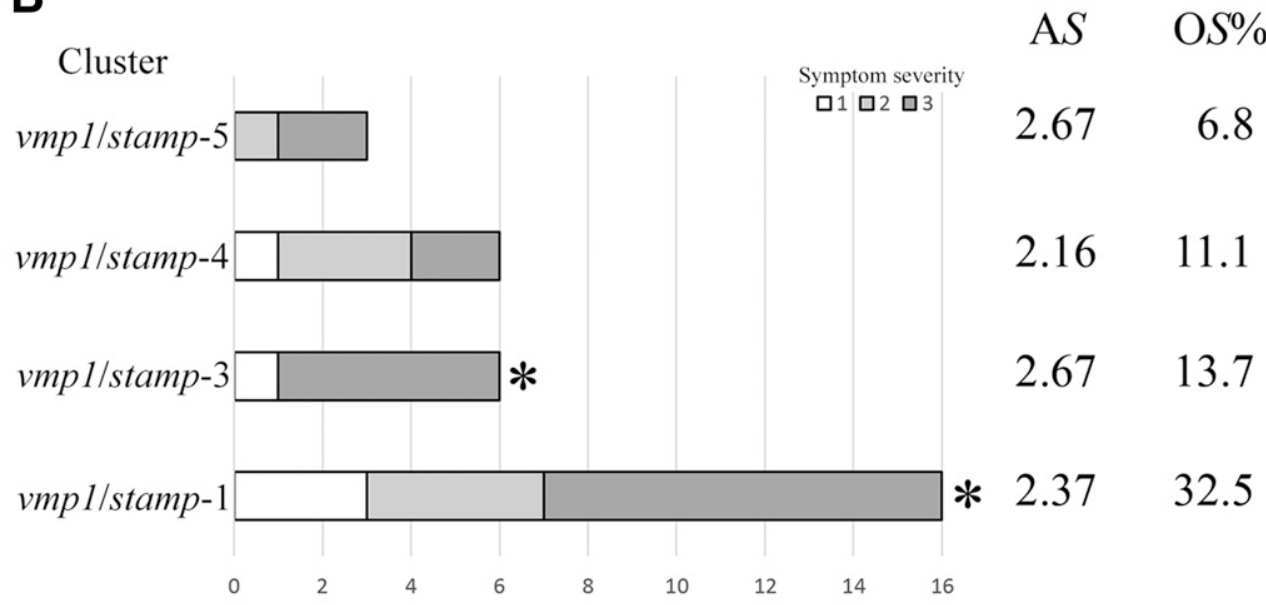

Number of Sangiovese plants infected by BNp in the study vineyard

Fig. 4. Phylogenetic position of vmpl/stamp types identified among bois noir phytoplasma (BNp) strains in the Chianti Classico area and their relationship with symptom and disease severity in Sangiovese grapevines. A, Unrooted phylogenetic tree inferred from vmp1 and stamp concatenated nucleotide sequences of 'Candidatus Phytoplasma solani' strains representing vmpl/stamp types in GenBank and identified in the Chianti Classico area (written in bold); minimum evolution method was carried out using the Jukes-Cantor model and bootstrap replicated 1,000 times. Names of strains are reported on the image. B, Graphic representing the distribution of symptom severity classes registered in 2016 in the study vineyard in association with BNp strains grouped according to vmpl/stamp phylogenetic clusters; significantly different distributions $\left(\chi^{2}\right.$ test, $\left.P<0.1\right)$ are indicated with an asterisk $(*)$ at the top of each bar. Average disease severity $($ A $S$ ) indicates the average severity of symptoms shown by grapevine plants infected by BNp strains grouped according to vmpl/stamp phylogenetic clusters and overall disease severity percentage (OS\%) indicates how much each BNp strains cluster contributes to the severity of the disease observed in the study vineyard in 2016. 
differences were observed in the distribution of $\mathrm{BNp}$ strains belonging to $v m p 1 /$ stamp phylogenetic clusters 4 (AS 2.16, OS\% 11.1) and 5 (AS 2.67, OS\% 6.8) (Fig. 4B).

The overall ratio between the nonsynonymous and synonymous mutations $(d N / d S)$ was $>1$ for both genes analyzed. In detail, for gene $v m p 1$, the $d N / d S$ test showed ratio $=2.482, P=0.014$; and for gene stamp, $d N / d S=2.368, P=0.019$. These results confirmed the high number of nonsilent $(d N)$ mutations revealed by sequence analyses.

\section{DISCUSSION}

Real-time PCR-based detection of GY phytoplasmas revealed that symptomatic grapevines observed in the vineyard examined in the present study were infected by BNp, excluding the presence of phytoplasmas associated with FD, previously reported in Tuscany (Belli et al. 2010). On the other hand, negative PCR results from symptomatic grapevines could be connected with the low abundance or sporadic distribution of phytoplasmas in symptomatic plant tissues (Constable et al. 2003).

Recent studies highlighted the fact that the impact of $\mathrm{BN}$ on vineyards and on the distribution of symptomatic grapevines is influenced by two main driving forces: the transmission of $\mathrm{BNp}$ from infected source plants to grapevines and the spontaneous remission of symptoms (possibly driving to recovery) of diseased grapevines (Mori et al. 2015; Murolo et al. 2014). Field symptom observations showed that BN impact on the examined Sangiovese vineyard, located in the Chianti Classico area of Tuscany, doubled from 2015 to 2016 (from 3.3 to $7.2 \%$ of symptomatic grapevines), indicating the high frequency of $\mathrm{BNp}$ transmission to grapevines as the main epidemiological driving force acting on $\mathrm{BN}$ incidence.

Molecular typing carried out by analyzing tufB, vmpl, and stamp gene nucleotide sequences revealed a great intraspecies strain diversity among BNp strains identified in symptomatic grapevines, the complexity of which increased over the 2 years. In detail, TaqMan real-time PCR assays differentiating the two main BNp tuf $B$ types present in Italy underlined the unique presence of $\mathrm{BNp}$ tuf $B$ type $b$ in the examined vineyard. This evidence is in accordance with data reporting the prevalence of $t u f B$ type $b$ in vineyards of central and southern Italy (Marchi et al. 2015; Murolo and Romanazzi 2015; Pacifico et al. 2007). Due to the main association of tufB types (a and b) with distinct epidemiological systems (Langer and Maixner 2004), it is reasonable to hypothesize that BNp ecology in the Sangiovese vineyard under study can be prevalently related to the host system $C$. arvensis $-H$. obsoletus $-V$. vinifera.

A deeper understanding of the degree of genetic variability among the BNp strains was obtained by the characterization of the $v m p l$ and stamp gene sequences. Based on RsaI-RFLP profiles of the $v m p l$ gene amplicons, three main $\mathrm{V}$ types were identified as widespread in both years (V11 and V12) or sporadic only in 2016 (V9). Previous studies reported the large presence of the type V12 in Tuscany, Piedmont, and Marche; V11 in Sardinia; and V9 in Sicily and Piedmont (Pacifico et al. 2009).

Based on sequence identity of $v m p l$ and stamp gene sequences retrieved from NCBI GenBank, it is possible to determine the presence of $80 \mathrm{vmpl}$ and 46 stamp genetic variants among ' $\mathrm{Ca}$. $\mathrm{P}$. solani' strains (Quaglino et al. 2016) (this study). Unexpectedly, in the examined Sangiovese vineyard in Tuscany, BNp strain populations showed more variability in gene stamp (16 sequence variants, of which 12 have been reported for the first time) compared with gene vmpl (12 sequence variants, of which 7 were reported for the first time in this study) nucleotide sequences.

In $\mathrm{BNp}$ strain populations identified in both years, the prevalent vmpl sequence variant (identified in $50 \%$ of the BNp strains) was vmFi1, identical to the sequence variant Vm43 (Quaglino et al. 2016), previously reported only in ' $\mathrm{Ca}$. P. solani' strains infecting Linaria vulgaris and $H$. obsoletus in Italy (Landi et al. 2015; Marchi et al. 2015). Phylogenetic analyses revealed that BNp strains of vmFi1 to vmFi12 sequence variants identified in Tuscany in this study grouped in clusters $v m p 1-1,-2$, and -3 along with previously reported sequence variants, including $\mathrm{BNp}$ and ' $\mathrm{Ca}$. P. solani' strains associated with bindweed-related host systems in Europe (Cimerman et al. 2009; Murolo and Romanazzi 2015; Pacifico et al. 2009; Quaglino et al. 2016).

In $\mathrm{BNp}$ strain populations identified in both years, the stamp sequence variants stFi1, stFi2, and stFi3 (identical to sequence variants St10, St5, and St18, respectively) were largely prevalent (identified in $85 \%$ of the BNp strains) (Table 4). Sequence variant St5 was already reported in central-eastern Italy (along with St18)

TABLE 5. Genetic diversity among vmp1/stamp types, identified in the Chianti Classico area, grouped in the same and in distinct phylogenetic clusters

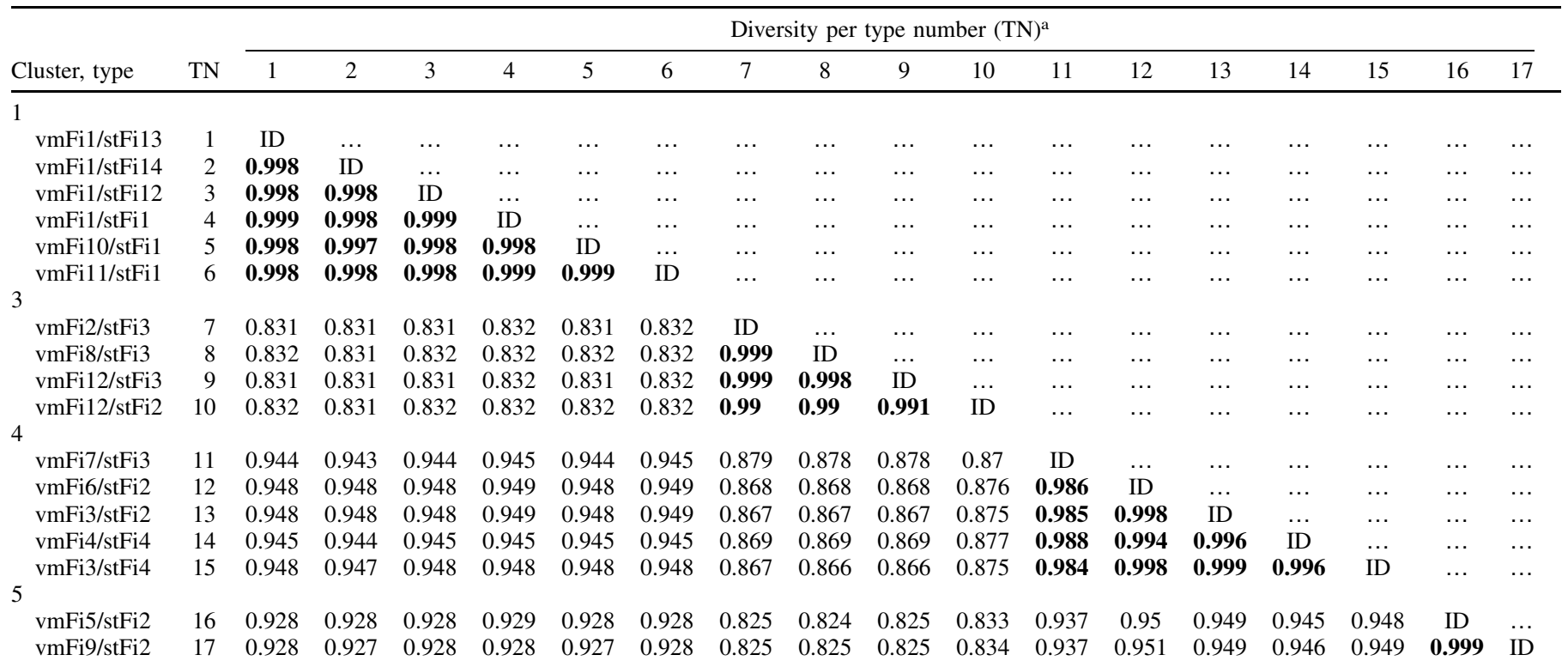

a Mean percent sequence identity within clusters: vmp1/stamp-1: 99.82; vmp1/stamp-3: 99.45; vmp1/stamp-4: 99.24; vmp1/stamp-5: 99.9. Mean percent sequence identity among clusters: vmp1/stamp-1 versus vmp1/stamp-3: 83.15; vmp1/stamp-1 versus vmp1/stamp-4: 94.67; vmp1/stamp-1 versus vmp1/stamp-5: 92.76 ; vmpl/stamp-3 versus vmp1/stamp-4: 87.09; vmpl/stamp-3 versus vmp1/stamp-5: 82.7; vmp1/stamp-4 versus vmp1/stamp-5: 94.61 . ID = 1 and numbers in bold indicate the diversity among vmpl/stamp types grouped in the same phylogenetic cluster. 
by Murolo and Romanazzi (2015) and widely spread in Slovenia (Fabre et al. 2011), Germany (Fabre et al. 2011; Johannesen et al. 2012), Austria (Aryan et al. 2014), and Macedonia (Atanasova et al. 2015). Intriguingly, sequence variant St10, the occurrence of which in symptomatic plants increased the most over the 2 years, was never reported in Italy before this study. In fact, previous studies reported St10 only on Solanum lycopersicum and H. obsoletus in the French Eastern Pyrenees (Fabre et al. 2011). Phylogenetic analyses revealed that $\mathrm{BNp}$ strains of stFi1 to stFi16 sequence variants identified in Tuscany in this study grouped in all three stamp clusters with previously reported sequence variants, including $\mathrm{BNp}$ and ' $\mathrm{Ca}$. P. solani' strains mainly associated with bindweed-related host systems in Europe (Cvrković et al. 2014; Fabre et al. 2011; Kosovac et al. 2015; Murolo and Romanazzi 2015; Quaglino et al. 2016).

As reported in previous studies (Durante et al. 2012; Quaglino et al. 2016), vmpl and stamp concatenated nucleotide sequences have been employed to improve the robustness of phylogenetic analyses. Within BNp strains infecting examined grapevines, a combination of vmpl and stamp sequence variants allowed the identification of $17 \mathrm{vmpl} / \mathrm{stamp}$ types grouped in clusters $\mathrm{vmpl/}$ stamp-1, $-3,-4$, and -5 . In detail, the prevalent type vmFi1/stFi1, consisting of the unreported combination of two known sequence variants present in Italy (Vm43) and the French Eastern Pyrenees (St10), grouped in cluster vmpl/stamp-1 with vmpl/stamp types identified in $H$. obsoletus, grapevine, and other host plants outside of Italy. The overall ratio between the nonsynonymous and synonymous mutations showed that vmpl and stamp genes in ' $\mathrm{Ca}$. $\mathrm{P}$. solani' strains in Tuscany are under a positive selection process. In contrast with previous studies reporting differences in intensive selection acting on vmpl and stamp genes (Murolo and Romanazzi 2015; Quaglino et al. 2016), the values of the $d N / d S$ ratio indicated a similar intensity of selection for both genes in $\mathrm{BNp}$ strain populations from Tuscany.

Two main hypotheses can be formulated to explain the surprising prevalent spread of the BNp vmp1/stamp type Vm43/St10 in the Chianti Classico area. First, such a BNp type was never detected before in Tuscany (no studies were previously carried out) but it was probably present in that ecosystem at least since 1997, when the Sangiovese vineyard was planted, and it coevolved, adapting to grapevines and other hosts. This hypothesis could be supported by the $d N / d S$ values of both $v m p l(2.482, P=0.014)$ and $\operatorname{stamp}(2.368$, $P=0.019)$ of $\mathrm{BNp}$ strain populations identified in the examined vineyard. In fact, these values are lower than those reported in previous studies for Euro-Mediterranean ' $\mathrm{Ca}$. P. solani' populations (vmp1: 4.637, $P=0.000$ and stamp: $2.711, P=0.004$ ) (Quaglino et al. 2016), possibly indicating a less intensive selection (higher level of adaptation) acting on $\mathrm{BNp}$ strains identified in the Sangiovese vineyard. Second, BNp vmp1/stamp type Vm43/St10, never reported before, could derive from plant materials or insects infected by ' $C a$. P. solani' strains harboring sequence variant $\mathrm{Vm} 43$ (present in Central Italy) and St10 (present in France) separately. Such strains, coinfecting the same hosts, may have undergone a recombination process leading to the appearance of this new $\mathrm{BNp}$ type in the Chianti Classico area. A similar recombination phenomenon was previously proposed for phytoplasmas of taxonomic group 16SrX (Danet et al. 2011).

Interestingly, in both years, Sangiovese clone grapevines exhibited a range of symptom severity, described by a symptomatic scale from 0 to 3 as proposed by Riedle-Bauer et al. (2010), with some modifications. Previous studies indicated that symptom severity of phytoplasma diseases can be influenced by four main factors: (i) environmental conditions (Hren et al. 2009; Murolo and Romanazzi 2015), (ii) phytoplasma abundance in infected plants (Martini et al. 2011), (iii) phytoplasma strain virulence (Seemüller and Schneider 2007; Seemüller et al. 2013), and (iv) plant host genotypes (Bisognin et al. 2008; Eveillard et al. 2016; Galetto et al. 2016; Quaglino et al. 2016; Roggia et al. 2014). Due to the genetic identity among clone Sangiovese grapevine plants and to the homogeneous environmental conditions in each year, the present study focused on investigating the possible correlation between the observed symptom severity and either the abundance or genotype of BNp strains.

Statistical analyses showed no significant correlation between relative abundance of BNp phytoplasma and symptom severity. On the other hand, statistically significant differences were observed in the distribution of BNp strains grouped in vmpl, stamp, and vmpl/ stamp phylogenetic clusters in grapevine plants showing symptom severity class 1,2 , and 3 . Considering the genetic homogeneity of grapevine plants within the examined vineyard, such evidence suggests that the genetic diversity of $\mathrm{BNp}$ strains could influence the symptom severity of infected grapevines. In particular, as reported for apple proliferation phytoplasmas (Seemüller and Schneider 2007; Seemüller et al. 2013), BNp strains grouped in distinct phylogenetic clusters could be associated with different ranges of virulence. Particularly, (i) BNp strains of the cluster vmp1/stamp-3, present mainly in grapevines showing symptom severity class 3 , have the highest $\mathrm{A} S$, suggesting a possible high virulence; and (ii) BNp strains of the cluster vmpl/stamp-1, present in grapevines showing symptom severity class 1,2 , and 3 , have an intermediate $\mathrm{A} S$ and the highest $\mathrm{O} S$, suggesting a possible moderate virulence. Regarding the prevalent (newly reported) BNp type Vm43/St10, its belonging to the cluster vmpl/stamp-1, combined with its widespread distribution in the examined vineyard, could lead to a hypothesis of its coevolution with the Sangiovese clone in the Chianti Classico area.

Fascinatingly, nucleotide sequence alignment of vmpl/stamp types identified in the Chianti Classico area revealed the larger intercluster genetic diversity of BNp strains (89.41\%, approximately 184 SNP distinguishing one strain from another) in comparison with the intracluster genetic diversity $(99.57 \%$, approximately 10 SNP distinguishing one strain from another). This evidence can suggest that, within each vmp1/stamp cluster, BNp strains with extremely similar Vmp1 and Stamp protein sequences could share analogous biological behavior and functions, leading also to possible differences in their virulence.

The extremely erratic scenario of host-pathogen-environment interactions involved in $\mathrm{BN}$ disease can lead to two main reasonable concerns regarding the association between symptom severity and BNp strains: (i) the variation of symptom severity and BNp strain in the same infected plant throughout years and (ii) the possible coinfection of the same plant by multiple $\mathrm{BNp}$ strains. Regarding the former, in this work, the accuracy of the analysis was improved by considering symptom severity and BNp strain type in the same season, as described in a previous study (Quaglino et al. 2016). Regarding the latter, the BNp strain coinfection in single grapevines was plausibly excluded by checking the collective length of the fragments composing the enzymatic digestion (RFLP) patterns of vmpl amplicons. In fact, through RFLP pattern visualization, a multiple phytoplasma strain infection can be detected by the copresence, in a single digested amplicon, of more than one pattern overlapped in the same electrophoretic lane. It is easily observed because the sum of the length of the pattern bands is higher than the amplicon size (Alma et al. 1996; Staniulis et al. 2000). In a previous study, the coinfection of grapevine plants by FDp strains was revealed using an approach based on the library analysis of $r p l V$-rps $C$ genes. It showed that, although multistrains infection is frequent, only one strain is strongly prevalent, representing over $99 \%$ of the population (Quaglino et al. 2010).

Despite the measures used to address these main concerns, further studies are needed to investigate in depth the experimental evidence collected in the present study concerning the possible virulence range among $\mathrm{BNp}$ strains according to their genetic background in other areas affected by BN. Moreover, epidemiological patterns of BNp strains, here identified in the Chianti Classico area, will be monitored in the coming years throughout European viticulture regions. 


\section{LITERATURE CITED}

Alma, A., Davis, R. E., Vibio, M., Danielli, A., Bosco, D., Arzone, A., and Bertaccini, A. 1996. Mixed infection of grapevines in northern Italy by phytoplasmas including 16S rRNA RFLP subgroup 16SrI-B strains previously unreported in this host. Plant Dis. 80:418-421.

Angelini, E., Bianchi, G. L., Filippin, L., Morassutti, C., and Borgo, M. 2007. A new TaqMan method for the identification of phytoplasmas associated with grapevine yellows by real-time PCR assay. J. Microbiol. Methods 68: 613-622.

Aryan, A., Brader, G., Mörtel, J., Pastar, M., and Riedle-Bauer, M. 2014. An abundant 'Candidatus Phytoplasma solani' tuf b strain is associated with grapevine, stinging nettle and Hyalesthes obsoletus. Eur. J. Plant Pathol. 140:213-227.

Atanasova, B., Jakovljević, M., Spasov, D., Jović, J., Mitrović, M., Toševski, I., and Cvrković, T. 2015. The molecular epidemiology of bois noir grapevine yellows caused by Candidatus Phytoplasma solani' in the Republic of Macedonia. Eur. J. Plant Pathol. 142:759-770.

Baric, S. 2012. Quantitative real-time PCR analysis of 'Candidatus Phytoplasma mali' without external standard curves. Erwerbs-Obstbau 54: 147-153.

Belli, G., Bianco, P. A., and Conti, M. 2010. Grapevine yellows in Italy: Past, present and future. J. Plant Pathol. 92:303-326.

Berger, J., Dalla Via, J., and Baric, S. 2009. Development of a TaqMan allelic discrimination assay for the distinction of two major subtypes of the grapevine yellows phytoplasma bois noir. Eur. J. Plant Pathol. 124:521-526.

Bisognin, C., Schneider, B., Salm, H., Grando, S., Jarausch, W., Moll, E., and Seemüller, E. 2008. Apple proliferation resistance in apomictic rootstocks and its relationship to phytoplasma concentration and simple sequence repeat genotypes. Bacteriology 98:153-158.

Chuche, J., Danet, J. L., Salar, P., Foissac, X., and Thiery, D. 2016. Transmission of 'Candidatus Phytoplasma solani' by Reptalus quinquecostatus (Hemiptera: Cixiidae). Ann. Appl. Biol. 169:214-223.

Cimerman, A., Pacifico, D., Salar, P., Marzachì, C., and Foissac, X. 2009. Striking diversity of $v m p 1$, a variable gene encoding a putative membrane protein of the stolbur phytoplasma. Appl. Environ. Microbiol. 75: 2951-2957.

Constable, F. E., Gibb, K. S., and Symon, R. H. 2003. Seasonal distribution of phytoplasmas in Australian grapevines. Plant Pathol. 52:267-276.

Cvrković, T., Jović, J., Mitrović, M., Krstić, Q., and Tosevški, I. 2014. Experimental and molecular evidence of Reptalus panzeri as a natural vector of bois noir. Plant Pathol. 63:42-53.

Danet, J. L., Balakishiyeva, G., Cimerman, A., Sauvion, N., Marie-Jeanne, V., Labonne, G., Laviňa, A., Battle, A., Križanac, I., Škorić, D., Ermacora, P., Serçe, Ç. U., Çağlayan, K., Jarausch, W., and Foissac, X. 2011. Multilocus sequence analysis reveals the genetic diversity of European fruit tree phytoplasmas and supports the existence of inter-species recombination. Microbiology 157:438-450.

Durante, G., Casati, P., Clair, D., Quaglino, F., Bulgari, D., Boudon-Padieu, E., and Bianco, P. A. 2012. Sequence analysis of S10-spc operon among $16 \mathrm{SrV}$ group phytoplasmas: Phylogenetic relationship and identification of discriminating single nucleotide polymorphisms. Ann. Appl. Biol. 161: 234-246.

Eveillard, S., Jollard, C., Labroussaa, F., Khalil, D., Perrin, M., Desquè, D., Pascal, S., Razan, F., Hévin, C., Bordenave, L., Foissac, X., Masson, J. E., and Malembic-Maher, S. 2016. Contrasting susceptibilities to Flavescence dorée in Vitis vinifera, rootstocks and wild Vitis species. Front. Plant Sci. 7:1762.

Fabre, A., Danet, J. L., and Foissac, X. 2011. The stolbur phytoplasma antigenic membrane protein gene stamp is submitted to diversifying positive selection. Gene 472:37-41.

Fialová, R., Válová, P., Balakishiyeva, G., Danet, J. L., Sâfárová, D., Foissac, X., and Navrátil, M. 2009. Genetic variability of stolbur phytoplasma in annual crop and wild plant species in South Moravia. J. Plant Pathol. 91:411-416.

Fisher, R. A. 1950. Statistical Methods for Research Workers. 11th ed. (rev.). Oliver and Boyd Press, Edinburgh, UK.

Foissac, X., Carle, P., Fabre, A., Salar, P., and Danet, J. L., and STOLBUREUROMED Consortium. 2013. 'Candidatus Phytoplasma solani' genome project and genetic diversity in the Euro-Mediterranean basin. Pages 11-13 in: Proc. 3rd Eur. Bois Noir Workshop, Barcelona, Spain. E. Torres, A. Laviña, and A. Batlle, eds.

Galetto, L., Miliordos, D. E., Pegoraro, M., Sacco, D., Veratti, F., Marzachì, C., and Bosco, D. 2016. Acquisition of Flavescence doreé phytoplasma by Scaphoideus titanus Ball from different grapevine varieties. Int. J. Mol. Sci. $17: 1563$.

Hall, T. A. 1999. BioEdit: A user-friendly biological sequence alignment editor and analysis program for Windows 95/98/NT. Nucleic Acids Symp. Ser. 41:95-98.

Hren, M., Nikolić, P., Rotter, A., Blejec, A., Terrier, N., Ravnikar, M., Dermastia, M., and Gruden, K. 2009. 'Bois Noir' phytoplasma induces significant reprogramming of the leaf transcriptome in the field grown grapevine. BMC Genomics 10:460.

Johannesen, J., Foissac, X., Kehrli, P., and Maixner, M. 2012. Impact of vector dispersal and host-plant fidelity on the dissemination of an emerging plant pathogen. PLoS One 7:e51809.

Kosovac, A., Radonjić, S., Hrnčić, S., Krstić, O., Toševski, I., and Jović, J. 2015. Molecular tracing of the transmission routes of bois noir in Mediterranean Vineyards of Montenegro and experimental evidence for the epidemiological role of Vitex agnus-castus (Lamiaceae) and associated Hyalestes obsoletus (Cixiidae). Plant Pathol. 65:285-298.

Kostadinovska, E., Quaglino, F., Mitrev, S., Casati, P., Bulgari, D., and Bianco, P. A. 2014. Multiple gene analyses identify distinct "bois noir" phytoplasma genotypes in the Republic of Macedonia. Phytopathol. Mediterr. 53: 491-501.

Landi, L., Riolo, P., Murolo, S., Romanazzi, G., Nardi, S., and Isidoro, N. 2015. Genetic variability of stolbur phytoplasma in Hyalesthes obsoletus (Hemiptera: Cixiidae) and its main host plants in vineyard agroecosystems. J. Econ. Entomol. 108:1506-1515.

Langer, M., and Maixner, M. 2004. Molecular characterisation of grapevine yellows associated phytoplasmas of the stolbur-group based on RFLP analysis of non-ribosomal DNA. Vitis 43:191-200.

Li, R., Mocka, R., Huangb, Q., Abadc, J., Hartungd, J., and Kinard, G. 2008. A reliable and inexpensive method of nucleic acid extraction for the PCRbased detection of diverse plant pathogens. J. Virol. Methods 154:48-55.

Maixner, M. 2011. Recent advances in bois noir research. Petria 21:95-108.

Marchi, G., Cinelli, T., Rizzo, D., Stefani, L., Goti, E., Della Bartola, M., Luvisi, A., Panattoni, A., and Materazzi, A. 2015. Occurrence of different phytoplasma infections in wild herbaceous dicots growing in vineyards affected by bois noir in Tuscany (Italy). Phytopathol. Mediterr. 54: 504-515.

Martini, M., Ermacora, P., Magris, G., Ferrini, F., and Loi, N. 2011. Symptom expression and 'Candidatus Phytoplasma prunorum' concentration in different Prunus species. B. Insectology 64:S171-S172.

Minguzzi, S., Terlizzi, F., Lanzoni, C., Poggi Pollini, C., and Ratti, C. 2016. A rapid protocol of crude RNA/DNA extraction for RT-qPCR detection and quantification of 'Candidatus Phytoplasma prunorum'. PLoS One 11: e0146515.

Mori, N., Quaglino, F., Tessari, F., Pozzebon, A., Bulgari, D., Casati, P., and Bianco, P. A. 2015. Investigation on 'bois noir' epidemiology in northeastern Italian vineyards through a multidisciplinary approach. Ann. Appl. Biol. 166:75-89.

Murolo, S., Mancini, V., and Romanazzi, G. 2014. Spatial and temporal stolbur population structure in a cv. Chardonnay vineyard according to vmpl gene characterization. Plant Pathol. 63:700-707.

Murolo, S., and Romanazzi, G. 2015. In-vineyard population structure of 'Candidatus Phytoplasma solani' using multilocus sequence typing analysis. Infect. Genet. Evol. 31:221-230.

Murolo, S., and Romanazzi, G. 2016. Multilocus sequence analysis as a powerful tool to monitor molecular epidemiology of 'Candidatus Phytoplasma solani’ at vineyard scale. Mitt. Klosterneuburg 66:40-73.

Nei, M., and Kumar, S. 2000. Page 333 in: Molecular Evolution and Phylogenetics. Oxford University Press, New York.

Nielsen, R. 2005. Molecular signatures of natural selection. Annu. Rev. Genet. 39:197-218.

Pacifico, D., Alma, A., Bagnoli, B., Foissac, X., Pasquini, G., Tessitori, M., and Marzachì, C. 2009. Characterization of bois noir isolates by restriction fragment length polymorphism of a stolbur-specific putative membrane protein gene. Phytopathology 99:711-715.

Pacifico, D., Foissac, X., Veratti, F., and Marzachì, C. 2007. Genetic diversity of Italian phytoplasma and French "bois noir" isolates. B. Insectology 60: 345-346.

Quaglino, F., Casati, P., and Bianco, P. A. 2010. Distinct $r p s C$ single nucleotide polymorphism lineages of Flavescence dorée subgroup 16SrV-D phytoplasma co-infect Vitis vinifera L. Folia Microbiol. (Praha) 55:251-257.

Quaglino, F., Maghradze, D., Casati, P., Chkhaidze, N., Lobjanidze, M., Ravasio, A., Passera, A., Venturini, G., Failla, O., and Bianco, P. A. 2016. Identification and characterization of new 'Candidatus Phytoplasma solani' strains associated with bois noir disease in Vitis vinifera L. cultivars showing a range of symptoms severity in Georgia, the Caucasus region. Plant Dis. 100:904-915.

Quaglino, F., Murolo, S., Zhao, Y., Casati, P., Durante, G., Wei, W., Bianco, P. A., Romanazzi, G., and Davis, R. E. 2017. Identification of new -J and -K 16SrXII subgroups and distinct single nucleotide polymorphism genetic lineages among 'Candidatus Phytoplasma solani' strains associated with bois noir in Central Italy. Australas. Plant Pathol. 46:31-34.

Quaglino, F., Zhao, Y., Casati, P., Bulgari, D., Bianco, P. A., Wei, W., and Davis, R. E. 2013. 'Candidatus Phytoplasma solani', a novel taxon associated with stolbur and bois noir related diseases of plants. Int. J. Syst. Evol. Microbiol. 63:2879-2894. 
Raudales, R. E., Stone, E., and McSpadden Gardener, B. B. 2009. Seed treatment with 2,4-diacetylphloroglucinol-producing pseudomonads improves crop health in low-pH soils by altering patterns of nutrient uptake. Phytopathology 99:506-511.

Riedle-Bauer, M., Hanak, K., Regner, F., and Tiefenbrunner, W. 2010. Influence of pruning measures on recovery of bois noir-infected grapevines. J. Phytopathol. 158:628-632.

Roggia, C., Caciagli, P., Galetto, L., Pacifico, D., Veratti, F., Bosco, D., and Marzachì, C. 2014. Flavescence dorée phytoplasma titre in field-infected Barbera and Nebbiolo grapevines. Plant Pathol. 63:31-41.

Schneider, B., Gibb, K. S., and Seemüller, E. 1997. Sequence and RFLP analysis of the elongation factor Tu gene used in differentiation and classification of phytoplasmas. Microbiology 143:3381-3389.

Seemüller, E., and Schneider, B. 2007. Differences in virulence and genomic features of 'Candidatus Phytoplasma mali', the apple proliferation agent. Phytopathology 97:964-970.
Seemüller, E., Sule, S., Kube, M., Jelkmann, W., and Schneider, B. 2013. The AAA plus ATPases and HfIB/FtsH Proteases of 'Candidatus Phytoplasma mali': Phylogenetic diversity, membrane topology, and relationship to strain virulence. Mol. Plant-Microbe Interact. 26:367-376.

Sharon, R., Soroker, V., Wesley, S., Zahavi, T., Harari, A., and Weintraub, P. 2005. Vitex agnus-castus is a preferred host plant for Hyalestes obsoletus. J. Chem. Ecol. 31:1051-1063.

Staniulis, J. B., Davis, R. E., Jomantiene, R., Kalvelyte, A., and Dally, E. L. 2000. Single and mixed phytoplasma infections in phyllody- and dwarfdiseased clover plants in Lithuania. Plant Dis. 84:1061-1066.

Tamura, K., Stecher, G., Peterson, D., Filipski, A., and Kumar, S. 2013. MEGA6: Molecular Evolutionary Genetics Analysis version 6.0. Mol. Biol. Evol. 30:2725-2729.

von Diest, S. G., Meitz-Hopkins, J. C., MacHardy, W. E., and Lennox, C. L. 2016. The effect of leaf shredding on apple scab in South African orchards. Plant Dis. 100:2094-2098. 